\title{
A Mobile Phone-based Context-aware Video Management Application
}

\author{
Janne Lahti, Marko Palola, Jari Korva, Utz Westermann*, Kostas Pentikousis and Paavo Pietarila \\ VTT Technical Research Centre of Finland, FI-90571 Oulu, Finland \\ e-mail: firstname.lastname @ vtt.fi \\ * University of California, Irvine, CA 92697-3425, USA \\ e-mail: westermann@acm.org
}

\begin{abstract}
We present a video management system comprising a video server and a mobile camera-phone application called MobiCon, which allows users to capture videos, annotate them with metadata, specify digital rights management (DRM) settings, upload the videos over the cellular network, and share them with others. Once stored in the video server, users can then search their personal video collection via a web interface, and watch the video clips using a wide range of terminals. We describe the MobiCon architecture, compare it with related work, provide an overview of the video server, and illustrate a typical user scenario from the point of capture to video sharing and video searching. Our work takes steps forward in advancing the mobile camera-phone from a video playback device to a video production tool. We summarize field trial results conducted in the area of Oulu, Finland, which demonstrate that users can master the application quickly, but are unwilling to perform extensive manual annotations. Based on the user trial results and our own experience, we present future development directions for MobiCon, in particular, and the video management architecture, in general.
\end{abstract}

Keywords: mobile camera phone application, mobile video management, video metadata and digital rights management

\section{INTRODUCTION}

Mobile phone manufacturers are increasingly adding new models with multimedia support and most modern medium- to high-end cell phones come with an integrated audio/video player, a camera to capture still and moving pictures, and some media editing software. The "coolness factor" fuels the popularity of mobile camera phones (MCP) and increases the volume of user-created media content. MCPs can record videos of up to several minutes, depending on the amount of memory available. Videos cannot be reasonably stored permanently on the mobile device due to the limited memory capacity available. Thus, users are in need of services that allow them to store their videos somewhere else, also because users want to create collections of their clips and share them with friends and relatives. Some will even opt for making part of their digital content available from a web site, or add it to their blog entries. Although none of these is news, nevertheless, there is very little automation in this process, and mobile applications for video management are not as commonplace as one might think despite the fact that MCPs have been on the market for quite some time.

The main problem of mobile content management is two-fold: how to automate permanent video clip storage, and how to do so in a way that is user-friendly, allows for easy clip lookups, and enables the user to share videos with others. Even though there are standardized ways to share videos over the cellular network, including the Multimedia Messaging Service (MMS) [1] and the upcoming IP Multimedia Subsystem (IMS) [2, 3], they must all be supported by the mobile operator, require infrastructure expenditures, and allow mainly for point-to-point video sharing. Moreover, a common characteristic of these services is that they do not include a solution for video management, and do not take full advantage of the context information available at the point of video capture. Our development effort takes a different approach and attempts to (a) enable the MCP to serve as an valuable video production tool in addition to being a video consumption channel, and (b) permit users to share videos with others irrespective of the level of network operator support for multimedia services.

Of course, one can argue that an MCP is not the ideal device for video management because of the limited CPU, memory and input/output capabilities, battery power consumptions considerations, and the diverse video formats and 
other technologies supported by the operating system. On the other hand, a MCP application can assist users to describe a video by associating it with annotations about, for example, where and when a video clip was shot, and who should be able to access it. The MCP application may have at its disposal different kinds of context information including sensors, a GPS device, a calendar service, and other Internet resources. All these are valuable sources for metadata that can be used to characterize the digital object and possibly uniquely identify it. Metadata plays a critical role in managing video content to the extent that Sarvas et al. [4] argue that it is not possible to manage media content effectively without them. The value of the metadata is illustrated in the following section, which presents a typical scenario where the user captures a video clip, associates it with metadata, and later searches for this particular video within her collection using an Internet-connected desktop PC. This paper details a solution to the problems related to user-created media content and annotation, and addresses some issues pertaining to sharing and storing videos using MCPs, and the challenges presented to the mobile video management system. The rest of this paper is organized as follows. Sections 3 and 4 discuss the implementation objectives of such video management system, and its architecture, respectively. Section 5 summarizes the results of a real user field trial evaluation, and Section 6 presents our current work in progress along with some future directions, which address certain shortcomings found in the first generation of the system. Finally, Section 7 reviews related work, and Section 8 concludes the paper.

\section{A TYPICAL USER SCENARIO}

We exemplify the use of the mobile video management system with a scenario involving a tourist in a sightseeing tour. The aim in this section is to provide an overall understanding of how the system works, essentially a high-level usercentered view of the requirements, before presenting, in the following section, the main objectives.

Figure 1 portrays Alice while on vacation in Oulu, Finland. Alice spends some time walking around the city center area, visiting several attractions, when she notices the Oulu cathedral clock tower from a distance. After approaching the church, she uses her MCP to record a short video of the cathedral, its clock tower, and the surrounding area. Alice uses MobiCon, an MCP application, which allows her not only to capture the video, but also to upload it to a server and notify her friends about recent findings during her tour of Oulu.
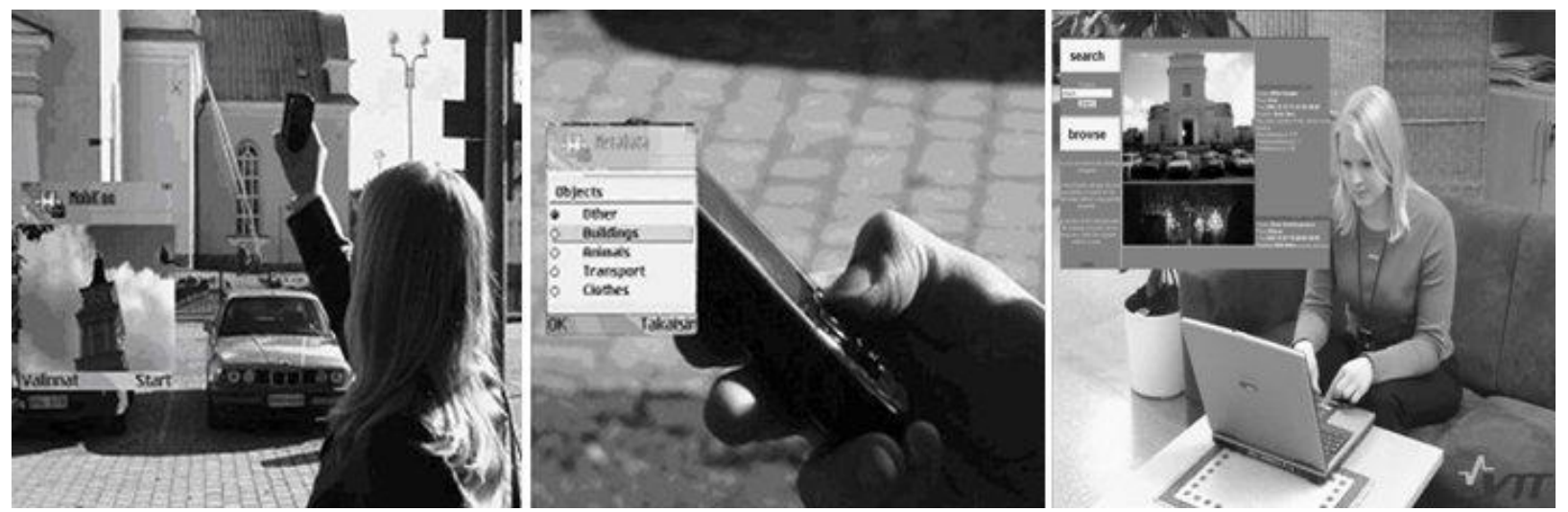

Figure 1: Alice records a video of the Oulu cathedral (left), annotates it with the term "Object > Buildings" (center), and later is able to search for it using the web interface of the Candela video management server (right).

After recording the video, Alice annotates it by selecting the prefedefined concepts "Holiday" and "Buildings" from the application metadata menu, and enters two keywords ("Church" and "Oulu") to describe the video clip more accurately. Alice saves the clip locally on the mobile phone memory card. Shortly afterwards, Alice decides to share the video with Bob, a friend of hers who is very interested in church architecture. With MobiCon, this is a simple process: Alice selects the video clip using a menu, chooses Bob from her contact list, and grants him the rights to watch the clip. She can subsequently upload the clip to the video server and MobiCon will automatically send a text message using the Short Message Service (SMS) to Bob with information on how to access the video. After receiving the text message, Bob can watch the video by opening its URL straight from his mobile phone.

After returning from her holidays, Alice can login to the video server using a web interface and access her large clip collection. Mobile videos tend to be short in length and relatively "focused" on a single theme. Unfortunately, Alice quickly finds herself having to deal with hundreds of videos with not-so-descriptive file names. Trying to locate the 
"church video" in the entire collection can quickly become tiresome and this is where video metadata annotation proves helpful. Although Alice is not likely to remember the exact time or date that the video was captured or uploaded to the video server, she does remember where she was and what the main theme of the video was. Searching for "Oulu" limits the set of possible videos and searching for "church" narrows the size of the search result set to a handful of clips. Once located, Alice can watch the movie clip delivered by the video management server in the most appropriate format for her platform.

\section{IMPLEMENTATION OBJECTIVES}

A mobile video management application has five main functions: video recording, metadata annotation, video storage, video sharing, and locating video clips in a collection. Any MCP application, such as MobiCon, should be robust and rich in functionality, yet easy to use and engaging, despite the restrictions imposed by the small display size and minimal keyboard. Moreover, application developers must pay attention to the way resources are used: network traffic should be minimized, battery power should be conserved when possible, and CPU and memory ought to be utilized with frugality. These restrictions come on top of the classic mobile phone application development nightmares (device incompatibilities, network application debugging, immature SDKs, and different operating system versions with undocumented bugs) making the development of an application like MobiCon challenging.

Video recording, the first function, is relatively straightforward to implement with vendor provided SDKs. However, the application should be robust during this phase and capable of handling critical events (including incoming phone calls and text messages). As illustrated in the previous section, video metadata annotation is necessary for searching stored clips in an efficient manner and is a central part of the design of MobiCon. We can identify three main issues that need to be addressed: (a) when shall the application collect the metadata, (b) which types of metadata should be stored so that locating the video will be easier later, and (c) how can the application acquire all this information in an elegant and robust manner. Practice indicates that, at least for home videos, the best time to annotate video clips is right after capturing them. However, at this point it is difficult to predict which information will be most valuable in locating the video later on. For example, for a resident of Oulu using MobiCon frequently while in town, the term "Oulu" will not be a good choice in order to discriminate between a large set of videos. For Alice, though, the same term will allow her to easily select all videos from her last visit to Northern Finland. Thus, one can argue that the best choice is to collect as much information as possible, and preferably do so in an automated way. On the other hand, this may lead to generating mostly low-level data or content features, such as exact dates and color histograms, which are neither easily recollected by most humans nor convey a direct meaning to them. Therefore, the application must allow the user to provide additional high-level information, and assist him in this by making the process as easy as possible.

The limited phone resources make video storage and video sharing particularly interesting problems to address. Permanent storage cannot be provided by the phone in a scalable manner: compared to other kinds of mass storage devices, such as hard drives, memory cards for mobile phones are still expensive and very limited in capacity. Users typically end up transferring their videos to their PC and then share them with others. This, of course, is not a truly mobile video management solution. An MCP application should use the network to store the videos and allow others to view them in an asynchronous manner. For video sharing, there are no universally supported media formats, and the device capabilities and the capacity of the access networks vary greatly. Because of this, the original video ought to be provided in several alternative formats using different encoding parameters. However, video transcoding is a computationally demanding process which cannot be performed in real time. Thus, alternative versions should be generated before the video can be retrieved, typically soon after a clip is uploaded. Furthermore, an important aspect in video sharing is the ability to control how the receivers use the shared video clips and limit redistribution, if necessary. One way of handling the video storage and sharing is to place the videos on a server. This way the MCP application needs to upload the video only once while remaining able to share it with an arbitrary number of recipients. The server can also take care of all security and computational intensive aspects of video storage, and enable the user to search for video clips later. In short, by making an MCP application directly pluggable to a searchable repository that takes advantage of the captured metadata leads to avoiding time-consuming and inefficient lookups of video clips based on filenames and file dates alone.

To sum up these requirements, MobiCon should be able to capture video clips using the internal mobile phone camera, assist the user to annotate each clip with metadata, store the clip to a remote video management server, permit him to share video clips with others and, last but not least, enable him to search large collections of video clips using a mobile phone, PC, or any other device with web access. Users should maintain full control of the entire video management chain, from source to destination, by tapping on a standards-compliant digital rights management (DRM) framework. 


\section{SYSTEM ARCHITECTURE}

The VTT Candela system, named after the European ITEA project CANDELA (Content Analysis, Networked Delivery and Architectures) was developed as a solution for general video management. It includes tools for video creation, analysis, annotation, storage, search and delivery phases. The Candela system was originally developed for the personal home video domain but, due to its modular and tiered architecture, it can be used as a basis for different video management applications by reusing some of the generic components and adding domain-specific ones. An important part of the entire architecture is the MCP application MobiCon, which can be used both on a standalone basis and as part of the entire system, as shown in Figure 2. Before delving in the details of the MobiCon architecture, we briefly introduce the Candela system; more details about the Candela architecture are given in $[5,6]$.

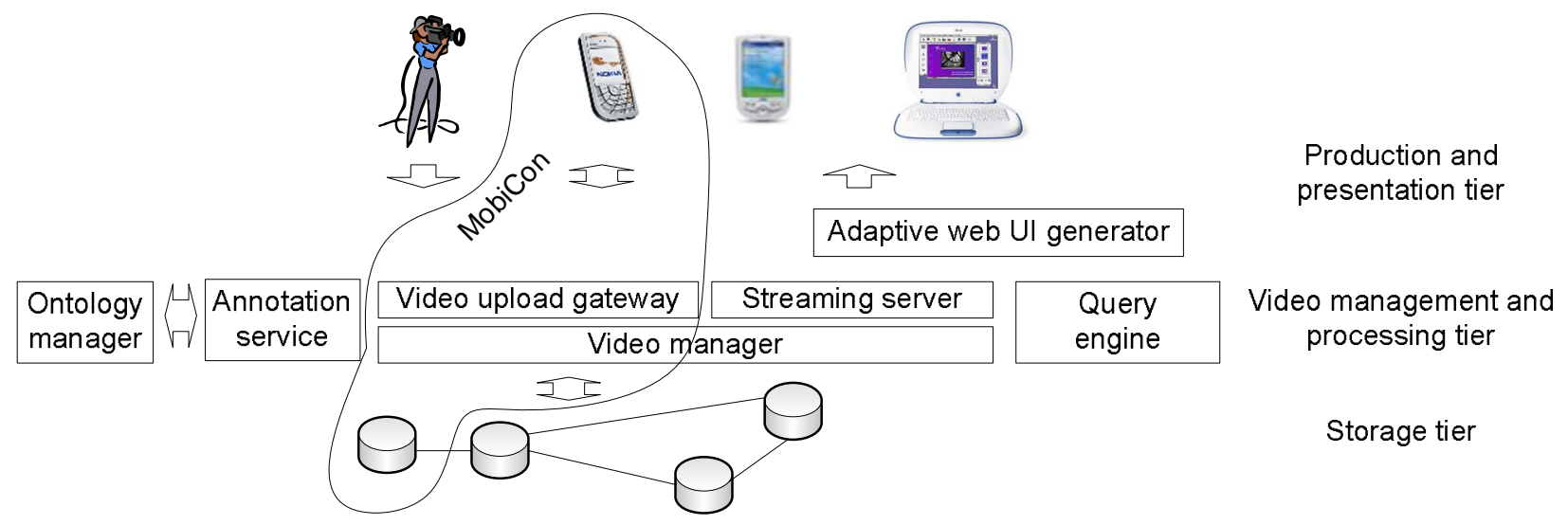

Figure 2: Candela and MobiCon system architecture.

\subsection{Candela}

Users can add video content to the Candela system produced by several different types of sources. The traditional method for home video content is to digitize analog content from a camcorder, or to use a digital one and upload the content to the system. Besides the home video domain, the Candela system can be a part of an enterprise-centered application such as, for example, an automatic security system handling the video content generated by a set of surveillance cameras. In the domain of personal mobile multimedia, the content is created in a mobile phone with video capture capabilities. In all application domains, the content is annotated automatically or semi-automatically, utilizing content analysis methods and input from sensors such as GPS positioning devices or context information which is available for example in a user's calendar. This information is captured in MPEG-7 metadata descriptions [7], which are stored along with the actual video data to support searching and managing video storage.

Candela uses the commercial Solid FlowEngine relational database system which provides scalability from a single in-memory database on a miniature embedded device to complex distributed and duplicated fault tolerant settings, which allows us to deploy more advanced configurations in the future. When storing, MPEG-7 descriptions are mapped to a relational schema so that SQL can be used for querying. In order to provide better results, the Candela query engine broadens user queries by suggesting additional search terms, which are, based on a domain ontology, closely related to the ones specified by the user or more descriptive. On the other hand, user profiles are used to restrict the amount of found matches to those that are the most relevant to the user.

Candela supports a very broad set of end user terminals ranging from cellular phones to high-end desktop computers. In order to provide a user friendly experience, the web-based interface dialogs are generated dynamically by using open source Apache Cocoon framework for XML transformations. This allows us to customize the amount of information presented to the user and the dialog between the user and the system to the capabilities of the used access device. Once the user has found an interesting video, a streaming video player is launched. This is either one of the off-the-self video players or Candela video browser which shows visualized metadata in addition to the video itself. The visualization allows the user to navigate through the video and find the relevant parts of it easily [5].

Providing optimal video quality for the end-user given the diversity of the source material, differences in user terminal capabilities and characteristics of networks, especially in the mobile domain, is a challenging task. MPEG-4 
standard offers some scalable video coding solutions, where the changing network capabilities for video delivery can be taken into account in real-time by inserting enhancement layers to the video stream in case of more available bandwidth. However, it was concluded in the project that the state-of-the art in scalable video coding does not offer reasonable quality as compared to the non-scalable stream at the same bitrate [8].

If the media source is a mobile phone with a low resolution camera, the need for transcoding is not as obvious as for more bandwidth consuming content, but overall we want media to be accessible across platforms and at the moment there are no uniformly supported video formats. Thus the solution was, at the expense of storage, to transcode the material to a representative set of formats and bitrates and develop a content negotiation plug-in for Helix streaming server in order to choose from those.

\subsection{MobiCon}

The MobiCon client-server architecture is shown in Figure 3. MobiCon consists of two different software components: the UploadClient, which is a mobile Java (J2ME) application running on a mobile phone and UploadGateway, which is implemented as a Java servlet in the Candela server. Architecture is based on the need to provide wireless access over a mobile phone network to enable storing video clips on the server where it is also possible to run more computationintensive operations such as video transcoding.

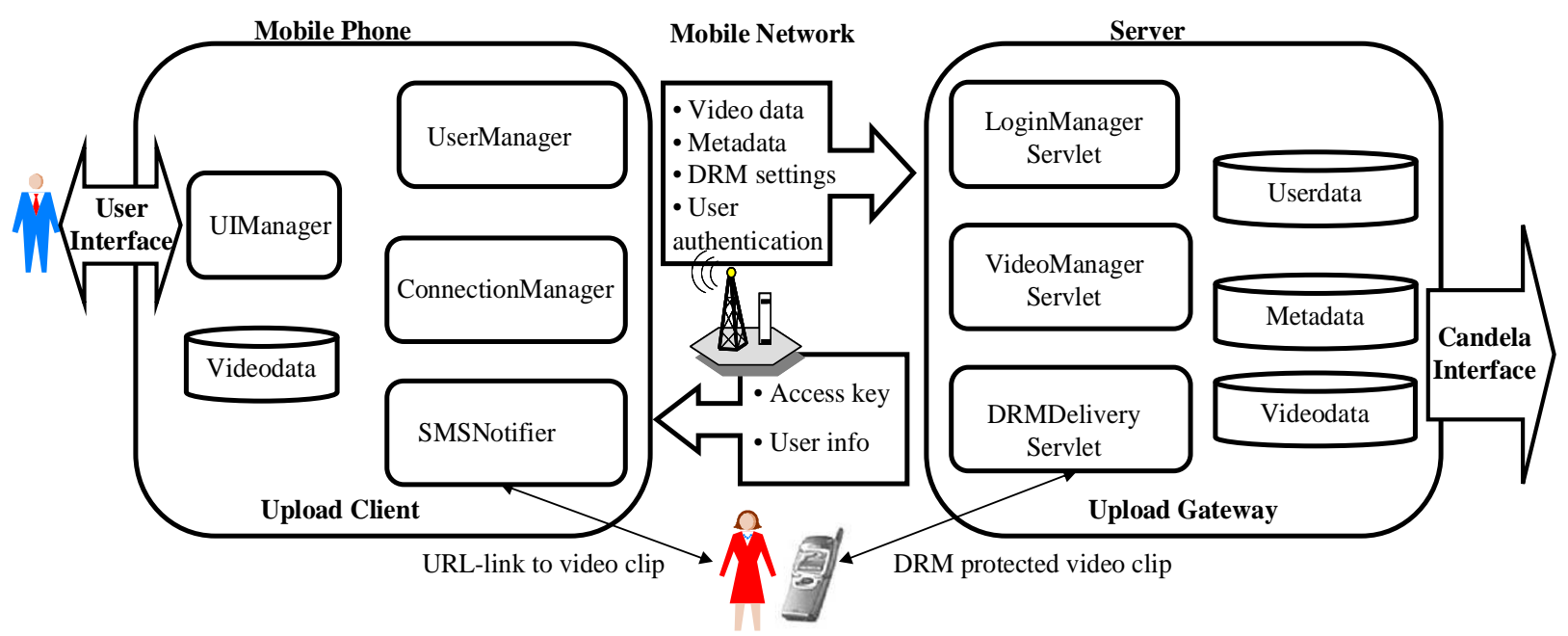

Figure 3: High-level description of MobiCon.

MobiCon naturally needs to be easily installed without any extra tools or additional instructions. The server allows distribution of MobiCon application easily to mobile phone users by using Over-The-Air (OTA) specification from the Open Mobile Alliance, which enables mobile applications to be downloaded and installed over the cellular network. After installation, the user is authenticated by the server using a username and password to log on the Candela system. The username and password are transferred to the UploadGateway and as a reply to successful authentication user profile information is transferred back to the UploadClient where UserManager stores user information (name, address, etc.), which are also used as metadata of captured video clips. The UploadClient no longer asks for username and password after the first time: for user convenience, it is assumed that the user stays the same after the first. Mobile phones are personal devices which are rarely lent to other people or left unguarded, the decision was made that the risk is not too large compared to the benefits achieved.

The UIManager is a controller component which is loaded first when the application is started. The UIManager coordinates the video capture using the mobile phone's camera, the saving of the video data to the Java Record Store system, and the sending of video sharing SMS messages to the other users. UIManager also provides user interfaces that are presented in the next Section. The ConnectionManager handles the connection between the UploadClient and UploadGateway providing data transfer using HTTP-protocol over the packet networks such as GPRS/EDGE/WCDMA. ConnectionManager delivers the captured video data, its metadata, user name, and DRM options to the Upload Gateway. If the user had chosen DRM protection for content sharing, the UploadGateway reply contains the identification keys to 
be shared with another user. The ConnectionManager creates a URL containing the received identification keys and returns them to the UIManager, which sends them to the other users via the SMSNotifier.

The UploadGateway serves multiple MobiCon users and provides access to the Candela system. Figure 3 presents an architectural overview of the UploadGateway. The LoginManager servlet handles all the tasks needed for user authentication on the server side. The VideoManager servlet takes care of all the functionalities receiving video data from the UploadClient to the UploadGateway. The received video and metadata descriptions are stored temporarily, the video clip is transcoded, a key frame picture is extracted from the video, and metadata is finally formatted to the MPEG7 XML format. All data including video clips, keyframes, and MPEG-7 are added to the database via Candela Interface.

The DRMDelivery servlet handles DRM video packaging and sharing. It is based on the Nokia Content Publishing Toolkit (NCPT) that allows creation of a DRM-protected video file package called Download Descriptor (DD), which holds the author, video name, size, and description. The DRMDelivery servlet creates a random number for DRM package name, which is delivered to other users in a URL address pointing to DRMDelivery servlet by SMS Notifier. The receiver of the shared video file needs only to open the URL link and to connect to DRMDelivery servlet using web browser in mobile phone and access the video clip if the DRM system in the mobile phone allows the access. The receiver can also see who the sender was and what kind of content is shared.

The MobiCon shared videos can be played directly via RealVideo player on mobile phone. With the DRM delivery channel a user can share video clips with other users who have a mobile phone supporting the OMA DRM standard and have a video player. DRM is an umbrella term for a set of technologies developed to protect against unauthorized copying and distribution of copyrighted material [9]. MobiCon is using DRM v1, although all mobile phones might not handle DRM protected content in standardized style [10].

\subsection{MobiCon Interface Flow Diagrams}

This section presents the MobiCon functionality from the user perspective with a walkthrough of typical usage scenarios. The user authentication and video capture is shown in Figure 4. The editing/uploading video clip is presented in Figure 5, which is expanded on Figure 6 and Figure 7 showing metadata annotation and DRM parameterization flows. Each figure comprises two parts: on the left side an Interface Flow Diagram [11] is given, while the right side presents the actual MCP screenshots.

User authentication and video capturing (Figure 4) starts with entering username and password for authentication with the server (Screenshots 1-2). If the username and password are valid, MobiCon receives the user's personal profile information and stores it in the phone memory. Then, MobiCon's main screen is displayed (Screenshot 3), where the user can choose to view and edit personal information, to load video clips, or to capture a new clip (Screenshot 4). A new video clip is captured in Capture Screen using Mobile Media API and it is recorded according to 3GPP specification using AMR coding for audio and H.263 at 176x144 pixels size at 15 frames per second for video. After a video is captured or loaded (Screenshot 5), the user is presented the Edit Screen (Screenshot 6) that ends the user authentication and video capturing scene.
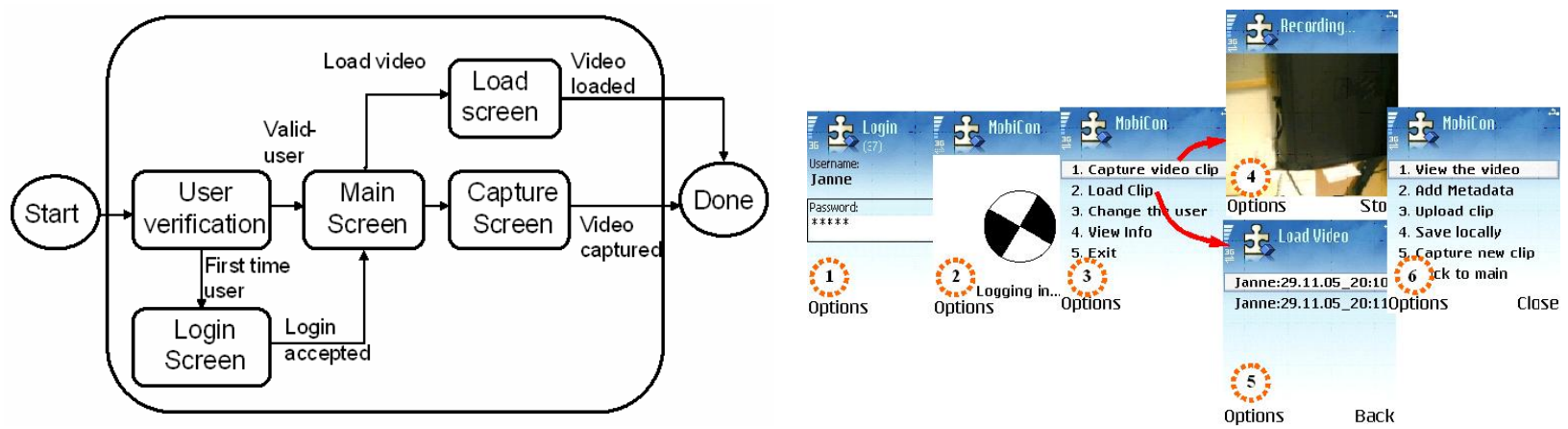

Figure 4: User authentication and video capturing: UI Flow Diagram (left) and UI screenshots (right).

Figure 5 presents the editing and uploading phase starting from the Edit Screen (Screenshot 1), which displays different editing options for the video clip. The user can choose to view the video clip in the Video View Screen (Screenshot 2), enter metadata annotations in the Metadata Screen (Screenshot 3), upload video clip (Screenshots 4, 5 and 6) either through the Access Screen or the DRM Screen, save, and capture a new video clip. 


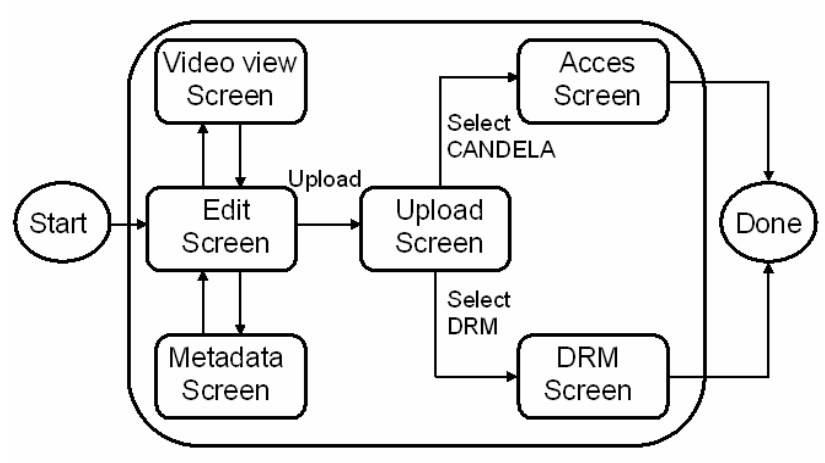

Figure 5: Editing and uploading the captured/loaded video clip: UI Flow Diagram (left) and UI screenshots (right).

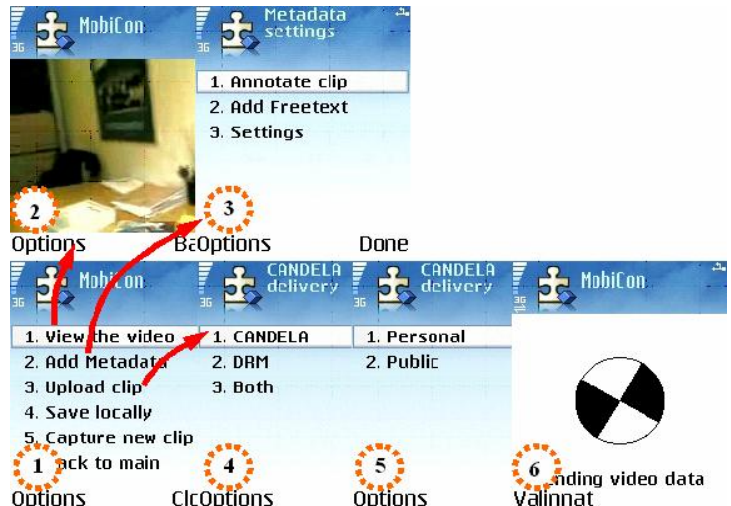

In the Edit Screen, the user may choose to upload the clip to the Candela server. The Upload Screen (Screenshot 4) prompts the user to choose the Candela or the DRM delivery channel or both. If the user chooses Candela delivery channel the video clip is uploaded to Candela server over mobile phone network and added to the Candela video database, from where the user can later search and access the video clip. A user must also choose whether the video clip is only for personal use or whether other Candela users can also find the clip via search. This is selected in the Access Screen (Screenshot 5). Then the video clip is uploaded via the Upload Gateway to the user video collection on the Candela server (Screenshot 6). In the server the video clip is handed over to the Video Manager Servlet, which transcodes the video clip into different formats and bit rates in order to provide a scalable service quality for different devices and network connections. Currently, the Video Manager Servlet prepares Real Video, H.264, and H.263 encodings for delivering the captured video content to mobile devices and MPEG-4 file format for desktop computers. In the future, scalable video codecs will remove the need of transcoding [12]. The DRM delivery channel (Screenshot 4), to prepare video clip for DRM protected sharing, is presented in details in Figure 7. The functionalities under the Metadata screen (Screenshot 3) are presented in Figure 6, which illustrates the flow diagram for the metadata annotation phase.
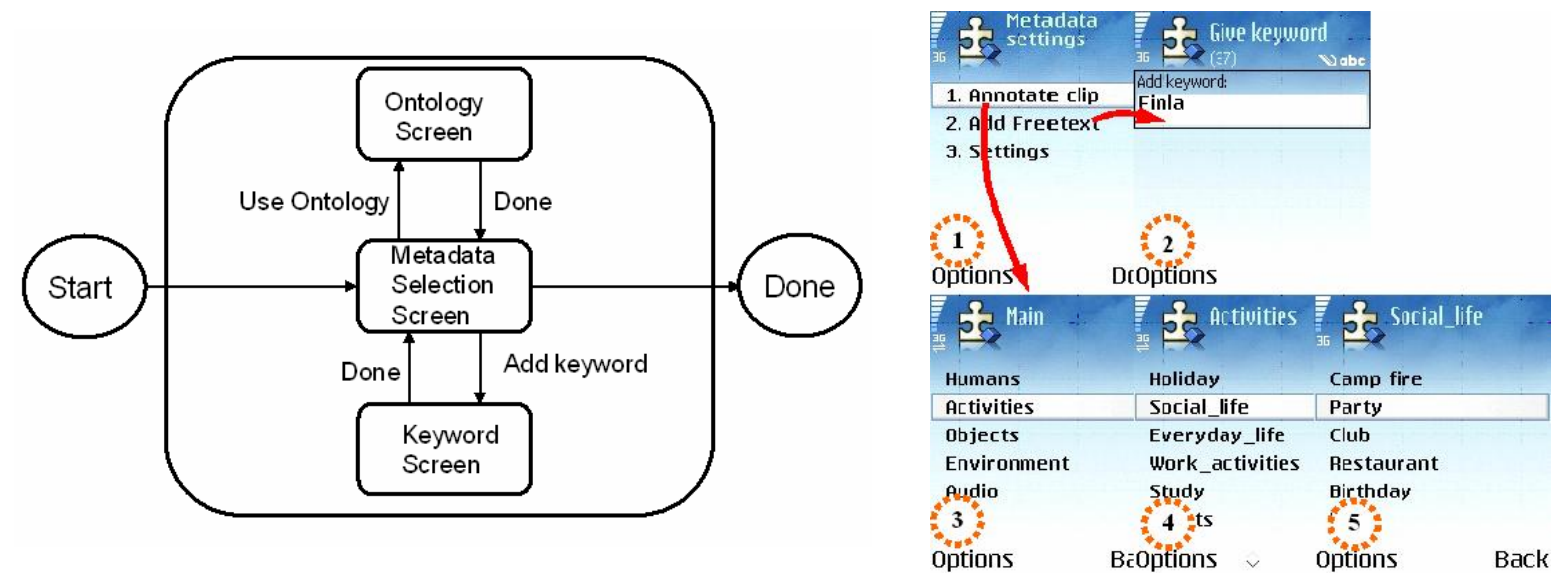

Figure 6: Giving metadata annotations: UI Flow Diagram (left) and UI screenshots (right).

The clip annotation starts from the Metadata Selection Screen (Screenshot 1) where the user has options to annotate clip using terms in a predefined domain ontology stored in the user's personal profile (Screenshots 3-5), add text descriptions by typing the nine-button keypad (Screenshot 2), and edit settings to update personal profile information, which is automatically added to the video clip metadata. When annotating via the Ontology Screen, the domain ontology can be accessed along a three-level hierarchical structure: the main level view (3) contains top-level concepts, which are then expanded in the sub-level menus $(4,5)$. All three levels are recorded to the clip. MobiCon also gathers context data that is available at the time of capture on the mobile phone such as user name, capture time, and duration of the clip. Additionally, MobiCon is able to connect to a GPS receiver supporting the NMEA protocol via Bluetooth. GPS provides 
longitude and latitude coordinates and a timestamp for the metadata. All the video clip metadata is uploaded with the video clip. In the final MPEG-7 file the context data includes the creator's name, region and country, date and time of day, GPS information, and length of the video clip as well as terms given by user and from the ontology tree are embedded in free text annotation elements the MPEG-7 provides.

Figure 7 presents the flow diagram for the DRM protected sharing phase. The starting point is the Delivery Method Screen (Screenshot 1) where the user adds the DRM option. The user is prompted by possible recipients from the mobile phone's contacts list (Screenshot 2). After selecting a receiver, the user can select between two DRM protection methods: Forward-Lock and Combined-Delivery (Screenshot 3). The Forward-Lock method prevents the receiver from resending the shared video clip from their mobile phones and it does not require additional configuration. The Combined-Delivery method (Screenshot 4) is used to specify access rights for the shared video clip. With the Combined-Delivery method, the user can set the dates the receiver can watch the video and how many times the video can be played by the receiver (Screenshot 5). After completing the Rights Selection Screen, the UploadClient sends the video, metadata and DRM information to the UploadGateway, which creates a DRM package at the server side and returns one identification key for each receiver. As soon as Upload Client receives the key(s) it generates and sends SMS messages to the receivers, who can download and play the video clip without MobiCon client software.
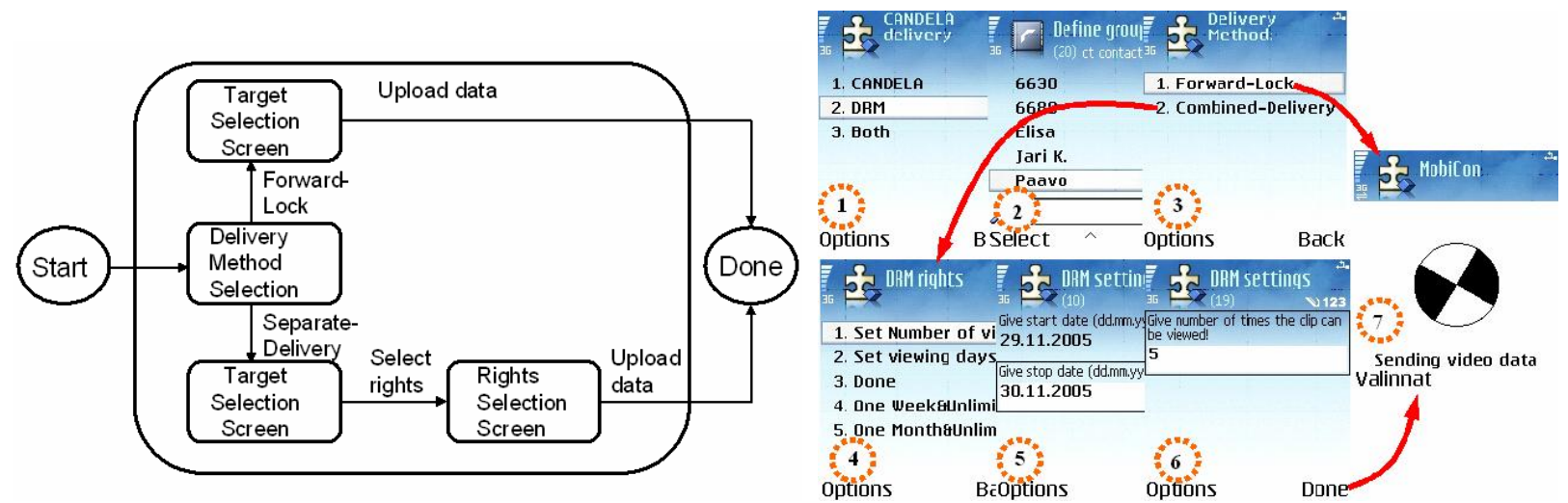

Figure 7: Sharing video DRM protected video clip: UI Flow Diagram (left) and UI screenshots (right).

\section{FIELD TRIAL EVALUATION}

Before initiating the field trial, MobiCon was tested extensively by a small group of developers/researchers from the Wireless Services group at VTT. Several issues were identified and fixed in the process, and we felt that both the application and the underlying video management service were ready for a field trial with real users. Due to space limitations, we will only summarize the results here; further details about the field trial are given in [13].

The main goal of the MobiCon field trial was to quantify application usability and assess its overall appeal to everyday users. The field trial lasted four weeks, starting on 14 February 2005 . We lent each of the ten trial participants a Nokia $6630 \mathrm{MCP}$ with MobiCon preinstalled. The primary author spent some time with each user introducing the application and explaining the main goals of the trial. At the end of the short tutorial, each participant had to take MobiCon for a "test ride" and continue using it for two weeks. Users were instructed to use the application as much as they would "in the real world". In other words, the aim of the trial was neither to stress- nor to beta-test MobiCon, but to replicate typical user behavior in a realistic environment; accordingly, participants were not forced to record, annotate, and upload as many videos as possible. During the trial period, we gathered data about the performance of the application and other context-related data, including user details, time and date; number of user interface (UI) selections made; session duration; type and number of metadata descriptions provided; and captured video duration, size, and upload bit rate. After the end of the trial period, users had to return the MCP and fill out an evaluation questionnaire.

The field trial results showed that MobiCon did overall meet all implementation objectives as specified in Section 3: users were able to capture video clips, annotate them with metadata, upload some of the clips to the video management server, and share clips with others. Both the application logs and the post-trial questionnaires indicated that MobiCon was relatively easy to use, even for inexperienced users. With regard to metadata annotation, most users agreed that an ontology-based term selection is a convenient way to add metadata to a clip, and it is preferred over free-text entries. Nevertheless, many users reported that the ontology tree structure and its size were far from ideal: some complained that 
the ontology tree structure was "too large", and that most terms were of little or no use; others wished the tree included more terms.

Despite the fact that most of the Oulu metropolitan area is covered by a fast 3G/UMTS network, seven out of ten users complained that the network was "slow". Moreover, the field trial revealed other issues including a couple of bugs which led to application crashes, and the concerns raised by some of the less technologically savvy participants about the security-related questions frequently asked by the MCP operating systems. The latter was due to the fact that MobiCon was not a signed application at the time of the field trial; the former were fixed before the time of this writing.

By and large, the participants were very satisfied with MobiCon (as an MCP application) and are looking forward to using such a service when it becomes commercially available. Even so, the field trial participants suggested several improvements, which, along with our own experiences, guide the evolution to the next phase of development.

\section{SYSTEM IMPROVEMENTS AND NEW DIRECTIONS}

Given the outcome of the field trial evaluation, we saw the imperative need to ease the annotation process by automation. One approach towards this problem would be to apply content analysis techniques on the video clips. Extracting highlevel annotations out of video content is already a challenging task in specialized domains with static and regular content such as sports or news $[14,15]$. With home video clips shot with today's camera phones, however, the task is even more daunting: home video content is highly irregular and diverse reflecting the varying interests of people; the technical skills of home videographers are rather limited resulting in shaky and blurry content; the still limited resolutions of phone cameras as well as high compression losses contribute to the bad content quality.

These problems led us to the different and much simpler approach of inferring reasonable annotation suggestions out of the rich context data about the clip capture that is available on mobile phones, as recently also suggested by [16]. For example, suggestions about the time of day, month, and season can be easily derived out of the system time on the phone; information about location of a clip capture can be obtained from the current cell ID or - with more modern phones or a GPS receiver accessible via Bluetooth - even the GPS position; given the location, one may access the user's address book to find out whether the clip was captured at a known location; given the time, one can look up possibly documented events in a calendar; etc.

Following this idea, we created an annotation web service for the creation of context-based annotation suggestions [6] and integrated it into the Candela system as outlined by Figure 8. With the creation of this web service, we pursued several goals:

Firstly, an annotation web service was painless to integrate with the already existing MobiCon application. After video capture, MobiCon merely needs to create an HTTP request with the context data and add the returned suggestions to the list of annotation keywords.

The second goal is extensibility. As it is easy to come up with further ideas of how to generate reasonable suggestions out of context, we want to be able to flexibly integrate new annotation methods as they become available. Therefore, we implemented the annotation web service on top of a plug-n-play bus architecture, which facilitates rapid integration of new methods for the generation of annotation suggestions. In addition, a centralized web service eliminates the logistic problem of deploying new annotation methods on the mobile clients.

Thirdly, the generation of annotation suggestions on a server relieved us from concerns with regard to the computational complexity of the generation of annotation suggestions. The highly parallelized architecture of the annotation bus further contributes to that aspect: all annotation suggestions are generated in parallel threads minimizing response times for each request and maximizing server utilization.

Figure 8 illustrates overview to the bus architecture of the annotation web service with the different modules currently implemented for the generation of annotation suggestions based on initial annotation.

In addition to adding the context-aware annotation web service, we also improved manual annotation of video clips. Because of the user complaints in the field trial with regard to the suitability of the predefined domain ontology for annotation to their personal situation and interests, we re-designed the system such that every user - excluding some general concepts forming the upper level of the hierarchy - can modify the ontology concepts and structure. Users can thus optimize their ontologies to their individual annotation needs, such that they can reach the concepts important to them in few navigation steps and without having to scroll through many irrelevant concepts on a small phone display on the way.

The individual ontologies of users are managed by an ontology manager on the Candela server. When a user logins to MobiCon, the application receives the personal ontology of the user from the ontology manager in RDF format and caches it for successive use along with the user's other profile data in the phone memory. 
We also have made other minor improvements to annotations. Now, MobiCon memorizes that last five keyword entries of the user and offers them for fast selection.

We believe that all these improvements will enhance the Mobicon user experience, making it easier, for the user, to annotate the videos with metadata. But we also see it is necessary to verify these improvements with a new field trial in a near future.

We have also further improvement ideas to the annotation process, which we are planning to integrate to MobiCon; for example, an even better metadata collection method suiting the nature of phone device could be audio annotation, so the user could add metadata descriptions to the videos just by speaking. Also typing metadata annotations, with ninebutton keypad, could be made easier by using dictionary assisted predictions for typed words.

With regard to the sharing of clips, MobiCon's current functionality is still limited, allowing the sharing of clips only right after capture. We are currently investigating the integration of a user interface into MobiCon that allows users to share any clip existing in their collections. In addition, the possibility of using other network connections, besides mobile networks, will be studied. Moreover, the remarks about slow cellular upload links, especially over GPRS, and the potential of high transfer fees in a public $3 \mathrm{G}$ network, prompted us to consider adding a peer-to-peer delivery channel as well. For example, users could connect to fixed networks by using network technologies like Bluetooth and WLAN, which can already be found on new mobile devices or they could use Bluetooth to share videos with user nearby. This is expected to satisfy a number of users who would prefer to capture several videos, annotate them on the spot, but upload them later.

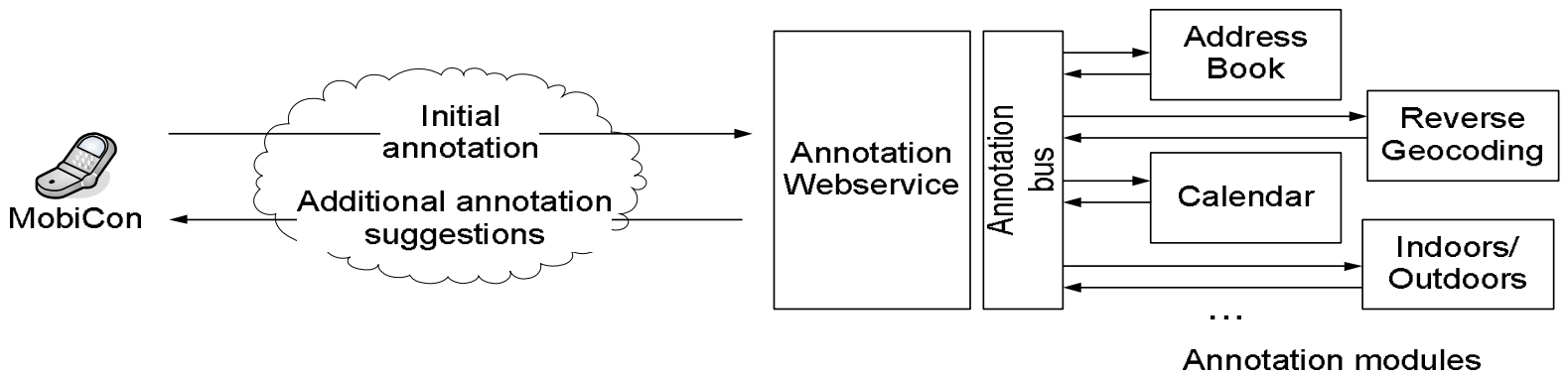

Figure 8: The Candela Annotation Web Service

\section{RELATED WORK}

Most of the existing mobile phone-based content management systems provide only capturing, annotating, and sharing of still photos, for example [4, 17]. Similar commercial applications as MobiCon exists such as Kodak Mobile (kodakmobile.com), Nokia's Movie Director (nokia.com), Nokia Album (nokia.com), and Nokia Lifeblog (nokia.com/lifeblog) and PhotoBlog (http://www.futurice.fi/PhotoBlog.html), but there is currently no similar mobile application that combines all video management features, i.e., video capturing, annotation using different context sources, sharing and storing videos for searching, into one [6, 13]. In general, existing commercial content sharing applications use different sharing methods such as MMS, email, blogs and even PC based tools for home personal video management [18].

Mobile video authoring applications exist for mobile phones without video sharing and DRM support, but they provide more advanced video editing tools in mobile device. For example, mProducer [19] provides video recording and editing in a mobile device, but it focuses on maximizing content capture space in a device and assisting the user in video editing, for example, by supporting shot detection and removing blurred frames. Video blogging using mobile devices is also becoming popular. FrameDrops [20] allows commenting captured video clips and provides video sharing via interactive web pages that align video clips over a map based on capture position. FrameDrops is targeted for work groups and online communities and they use Flash video format. MobiCon and Candela system targets personal video management by supporting video search in both mobile and desktop environments allowing sharing in different video formats.

Resent video annotation research is more focused on automatically summarizing video data from existing MPEG-7 video descriptions [21, 22] and enhancing the results using a query-based video personalization and summarization to show a shortened video summary of a large video content [23]. Also, some applications exist that automatically gather 
video metadata from a specific type of video content, for example news broadcasts. IBM [23] has also developed system for creating personalized video content from video databases for PDA and PC users. We used similar technologies for example, MPEG-7 metadata files, but the main differences to MobiCon and video server are that we support DRM to control video redistribution, we only use high-level features (objects, person names, situation, address and town names) to annotate the video clips in MPEG-7 file and we need to transcode video clips for streaming both PC and mobile devices. We also did not implement shot boundary detection in MobiCon because mobile videos are short and they typically consist of a single shot. More about mobile video streaming can be found in [21].

A mobile network-based video sharing service is already commercially launching by mobile operators such as Telecom Italia Mobile and TLC in Hong Kong. In telecommunication networks, a video sharing service is based on IMS and it allows sharing real-time or pre-recorded video clips during normal voice calls using a separate data call. Both parties hear each other and watch the same video until they end the video sharing call [3]. MobiCon type of tools will benefit of IMS system, since IMS allows point-to-point access between mobile phones and it can replace SMS-based content delivery. MobiCon only uses a GPRS packet connection to transmit video data to video server and users are not point-to-point connected and sharing is not real-time. We expect that similar applications as MobiCon will be available also as IMS services managed by mobile operators.

Finally, other technologies such as sensors in mobile phone [19] and RFID tags and readers [24] will be available to provide alternative ways to users to access shared content.

\section{CONCLUDING REMARKS}

This paper introduced a video management solution, which has at its core MobiCon, a mobile camera phone application. We illustrated a typical scenario involving a tourist creating short videos while on a sightseeing tour, laid out our original implementation objectives, described the overall system architecture, and detailed the main features of the application using Interface Flow Diagrams and application screenshots. After summarizing the results of the first MobiCon field trial, we presented a set of improvements to the original implementation of the application, and gave a short account of our latest development efforts. Finally, this paper compared MobiCon with other related research and development efforts and pointed out significant differences, stating possible future directions.

Although MobiCon has reached a certain level of maturity, there are many issues that still remain to be tackled. Certain security concerns regarding video clip access for invited receivers, along with improvements on the digital rights management component, top the list. Once these have been addressed, the next step should be to open up the video management server to a larger group of users and allow them to use it in combination with MobiCon. This will permit us to evaluate the complete service under a much larger and diverse set of configurations, which ideally should include users in other countries and, hopefully, in other continents as well. Porting MobiCon to more platforms is also in our current plans, as is pursuing business development opportunities for we seek closer cooperation with mobile network operators and startup video management service providers in order to create a commercially-available turnkey solution. To that end, we are exploring alternative user scenarios, which are not restricted to the home video market segment. Finally, depending on user interest, we are considering adding support for short-range, peer-to-peer communication using Bluetooth and WiFi.

On the research front, we are exploring how content analysis methods can be integrated with the annotation web service. Although the system architecture has been designed to be extensible from the ground up, it is still not meaningful to implement annotation suggestion modules based on content analysis methods, because the video would have first to be uploaded and analyzed by the server in order for the annotation web service to provide suggestions. The resulting delay in the annotation process may be prohibitive. Perhaps the solution can come from a distributed version of the annotation bus, which allows us to keep annotation suggestion modules on the MCP. This will also address some privacy concerns arising from the need to have certain personal context data, such as address book contacts and calendar entries, transferred over the cellular network to the web service.

\section{ACKNOWLEDGEMENTS}

MobiCon and Candela were developed as part of the EUREKA/ITEA CANDELA (Content Analysis, Networked Delivery and Architectures) project during 2004-2005. Funding provided by TEKES National Technology Agency of Finland, and VTT Technical Research Centre of Finland is gratefully acknowledged. 


\section{REFERENCES}

1. L. Novak and M. Svensson, "MMS - Building on the success of SMS", Ericsson Review, 78(3), pp. 102-109, 2001.

2. J.F. Huber, "Toward the mobile Internet", Computer, 35(10), pp. 100-102, 2002.

3. Nokia, "White Paper: Video Sharing v1.0", Forum Nokia, 2005.

4. R. Sarvas, E. Herrarte, A.Wilhelm, and M. Davis, "Metadata creation system for mobile images", Proceedings of International Conference on Mobile Systems, Applications, and Services (ACM MobiSys), pp. 36-48, ACM Press, Boston, Massachusetts, USA, 2004.

5. A. Sachinopoulou, S-M. Mäkelä, S. Järvinen, P. Pietarila, J. Peltola, and M. Jurvansuu, "Personal Video Retrieval and Browsing for Mobile Users", Multimedia on Mobile Devices, Proceedings of SPIE-IS\&T Electronic Imaging, 5684, pp. 219-230, 2005.

6. J. Lahti, U. Westermann, M. Palola, J. Peltola, and E. Vildjiounaite, "Context-Aware Mobile Capture and Sharing of Video Clips", Handbook of Research on Mobile Multimedia, I. K. Ibrahim \& J. Kepler (eds.), Idea Group, Hershey, Pennsylvania, USA, 2006, to appear.

7. B. S. Manjunath, P. Salembier, and T. Sikora (Eds.), Introduction to MPEG-7: Multimedia Content Description Language, John Wiley \& Sons, New York, New York, USA, 2002.

8. J. Vehkaperä and J. Peltola, "Optimized Decoding Scheme for Erroneous MPEG-4 FGS Bitstream", Proc. of IEEE International Symposium on Circuits and Systems 2005 (ISCAS2005), Kobe, Japan, 2005.

9. W. Jonker and J.-P. Linnartz, "Digital Rights Management in Consumer Electronics Products", IEEE Signal Processing Magazine, 21(2), pp. 82-91, 2004.

10. J. Irwin, "Digital Rights Management: The Open Mobile Alliance DRM specifications", Information Security Technical Report, 9(4), pp. 22-31, 2004.

11. S. Ambler, The Object Prime $3^{\text {rd }}$ Edition, Agile Model Driven Development with UML 2, Cambridge University Press, 2004.

12. A. Vetro, C. Christopoulos, and H. Sun, "Video transcoding architectures and techniques: An overview", IEEE Signal Processing Magazine, 20(2), pp. 18-29, 2003.

13. J. Lahti, K. Pentikousis, and M. Palola, "MobiCon: Mobile video recording with integrated annotations and DRM", Proceedings of IEEE Consumer Communications and Networking Conference (IEEE CCNC 2006), Las Vegas, Nevada, USA, 2006, to appear.

14. P. Duygulu, J. Pan, and D.A. Forsyth, "Towards Auto-Documentary: Tracking the Evolution of News Stories", Proceedings of ACM Multimedia, pp. 820-827, New York, New York, USA, 2004.

15. H. Xu and T. -S. Chua, "The Fusion of Audio-Visual Features and External Knowledge for Event Detection in Team Sports Video", Proceedings of the 6th ACM SIGMM International Workshop on Multimedia Information Retrieval (MIR), pp. 127-134, New York, New York, USA, 2004.

16. M. Davis, S. King, N. Good, and R. Sarvas, "From Context to Content: Leveraging Context to Infer Multimedia Metadata", Proceedings of ACM Multimedia, pp. 188-195, New York, New York, USA, 2004.

17. M. Davis, "Mobile Media Metadata: Metadata Creation System for Mobile Images", Proceedings of ACM Multimedia, pp. 425-445, New York, New York, USA, 2004.

18. Y. Wang, P. Zhao, D. Zhang, M. Li, and H. Zhang, "MyVideos - A System for Home Video Management", Proceedings of ACM Multimedia, pp. 412-413, Juan Les Pins, France, 2002.

19. C.-M. Teng, C.-I. Wu, Y.-C. Chen, H. Chu, and J. Y. Hsu, "Design and Evaluation of mProducer: A mobile authoring tool for personal experience computing", Proceedings of the third International Conference on Mobile and Ubiquitous Multimedia (MUM), pp. 141-148, College Park, Maryland, USA, 2004.

20. T. Gross and M. Kleppe, "FrameDrops: a mobile VideoBlog for workgroups and virtual communities", Proceedings of International Conference on Supporting Group Work, pp. 128-131, Sanibel Island, Florida, USA, 2005.

21. M. Etoh and S. Sekiguchi, "MPEG-7 enabled digest video streaming over 3G mobile network", Proceedings of the 12th International PacketVideo Workshop, Pittsburgh, Pennsylvania, USA, 2002.

22. P. M. Fonseca and F. Pereira, "Automatic video summarization based on MPEG-7", Signal Processing: Image Communication, 19(8), pp. 685-699, 2004.

23. B. L. Tseng, C.-Y. Lin, and J. R. Smith, "Video personalization and summarization system for usage environment", Journal of Visual Communication and Image Representation, 15(3), pp. 370-392, 2004.

24. Y.-M. Cheng, W. Yu, and T.-C. Chou, "Capturing context: Life is sharable: blogging life experience with RFID embedded mobile phones", Proceedings of the 7th International Conference on Human Computer Interaction with Mobile Devices and Services (MobileHCI), pp. 295-298, Salzburg, Austria, 2005. 